\title{
Detection and Classification of Brain Tumor Based on Multilevel Segmentation with Convolutional Neural Network
}

\author{
Rafiqul Islam, Shah Imran, Md. Ashikuzzaman, Md. Munim Ali Khan \\ Department of Computer Science and Engineering, Dhaka University of Engineering \& Technology, Gazipur, \\ Bangladesh
}

Correspondence to: Rafiqul Islam, rafiqul.islam@duet.ac.bd; Shah Imran, shahimrancoxbd@gmail.com; Md. Ashikuzzaman, ashik0172578@gmail.com; Md. Munim Ali Khan, munimalikhan90@gmail.com Keywords: Segmentation, Classification, Non-Cancerous Tumor, Cancerous Tumor, Feature Extraction, Convolutional Neural Network

Received: April 1, $2020 \quad$ Accepted: April 27, $2020 \quad$ Published: April 30, 2020

Copyright $\odot 2020$ by author(s) and Scientific Research Publishing Inc.

This work is licensed under the Creative Commons Attribution International License (CC BY 4.0).

http://creativecommons.org/licenses/by/4.0/

\section{(c) (1) Open Access}

\section{ABSTRACT}

Magnetic Resonance Imaging (MRI) is an important diagnostic technique for early detection of brain Tumor and the classification of brain Tumor from MRI image is a challenging research work because of its different shapes, location and image intensities. For successful classification, the segmentation method is required to separate Tumor. Then important features are extracted from the segmented Tumor that is used to classify the Tumor. In this work, an efficient multilevel segmentation method is developed combining optimal thresholding and watershed segmentation technique followed by a morphological operation to separate the Tumor. Convolutional Neural Network (CNN) is then applied for feature extraction and finally, the Kernel Support Vector Machine (KSVM) is utilized for resultant classification that is justified by our experimental evaluation. Experimental results show that the proposed method effectively detect and classify the Tumor as cancerous or non-cancerous with promising accuracy.

\section{INTRODUCTION}

Magnetic Resonance Imaging (MRI) is a wonderful tool or technique to visualize inside the human body with amazing clarity. It offers much greater contrast between the diverse soft-tissues of the body than compared to the X-ray or Computed Tomography (CT) which involves doses of ionization radiation. Also, the detailed images can be produced by the MRI technique in any direction. Clinically, radiologists qualitatively analyze films produced by MRI which provides rich information about the human soft-tissue anatomy. Particularly, MRI is useful in accordance with neurological (brain), musculoskeletal and onco- 
logical imaging. Therefore, without any harmful radiation, it is regularly used for detection of Tumors, lesions, and other abnormalities in soft-tissues. Doctors and surgeons analyze complex diseases like brain Tumors using an MRI image that enables the surgeon to detect and solve the problem relating to these diseases [1]. Image classification is an emerging field to computer-assisted diagnosis in brain MRI Tumors classification.

Different classification methods have been developed over the last few years. Most of the methods involve segmentation, feature extraction and classification process to classify the Tumors [2] in which binary thresholding, Principle Component Analysis (PCA) [3] and Support Vector Machine (SVM) classifier [4] methods are widely used as segmentation, feature extraction, and classification methods, respectively. As an example, binary thresholding with discrete wavelet transformation (DWT) followed by PCA, and SVM was combined to develop an image classification method that was used to detect normal and abnormal brain [5] and cancer detection [6]. An improvement of this method was developed considering kernel SVM (KSVM) instead of SVM in [7] for abnormality detection of brain MRI. Highlighting the benefits of $\mathrm{k}$-means clustering and PCA based segmentation method for brain MRI was described and developed in $[8,9]$. CNN has been recently used in many fields. Particularly in the field of image classification and recognition, CNN was applied for classification [10], segmentation [11], and so on [12]. From the literature, it is seen that since the use of basic thresholding method contains unwanted artifacts or objects specially for MRI, improving the segmentation can improve the overall accuracy of the classification process.

The objectives of the paper are to segment the Tumor from brain MRI properly and classify the Tumor as cancerous or non-cancerous brain Tumor with better accuracy. Benign or non-cancerous brain Tumor diseases are Alzheimer's, Huntington, Pick and Alzheimer with Agnosia whereas malignant or cancerous brain Tumor diseases are Glioma, Meningioma, Metastatic, and Sarcoma. In this work, an efficient classification method is used to identify the Tumor as cancerous or non-cancerous in which multilevel segmentation (MLS) method and CNN are developed to segment and extract features, respectively for the classification process. This method is named as MLS-CNN throughout the paper. The residue of the paper is organized as follows: In Section 2, the methodology of our work is explained and Section 3 is devoted to describing the experimental evaluation of the algorithm. Finally, our conclusion is presented in Section 4, respectively.

\section{METHODOLOGY}

For the purposes of classification of Tumor as cancerous or non-cancerous from brain MRI, the proposed MLS-CNN classification method consists: preprocessing, clustering, segmentation, feature extraction, and classification process. Preprocessing is the first stage in image analysis process which is used to smoothing, sharpening and reducing the noise of input; Segmentation is the basic stage of the classification system to separate the targeted objects; once the interesting objects are isolated from the input, certain features are made, and these are used to classify the objects into particular classes. The working procedure of the proposed MLS-CNN model is shown in the Figure 1.

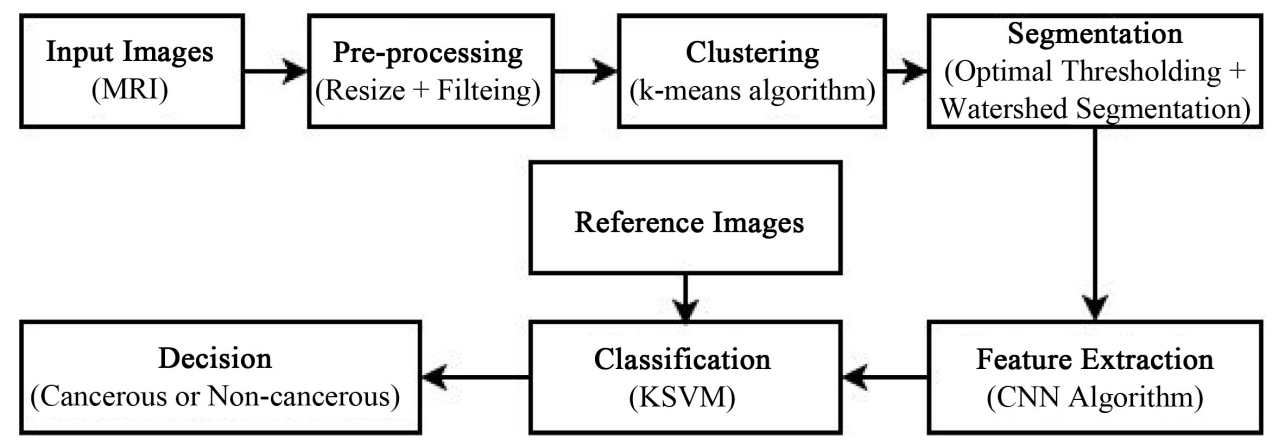

Figure 1. Block diagram of proposed MLS-CNN algorithm for Tumor detection and classification. 


\subsection{Preprocessing}

Image preprocessing technique is the first step of the method that is used to improve the quality of an image before processing it into an application. The aim of image preprocessing is to remove noise from the original image and for this, we used Gaussian, high pass and median filtering techniques. Gaussian filter is implemented to reduce the noise present in MRI brain images; High pass filtering removes unwanted frequencies from the image, and Median filtering increases the image quality.

\subsection{Clustering}

Clustering is the task of categorizing the objects into several groups in such a way that objects in the same group are more homogeneous to each other than to those in other groups. For clustering, k-means algorithm is the most popular and widely used method to categorize object in a group due to its efficiency and simplicity [13]. It is an iterative technique to find local maxima in each iteration. In k-means clustering process, each group is represented by one prototype object, and a new data is assigned to the nearest prototype and therefore to that cluster. The iterative process to calibrate the placing of the prototypes is performed as below:

- select objects randomly from the training data set, which becomes the prototypes;

- assign other objects to the nearest prototype;

- calculate the new prototype so that the mean of all objects having the same level;

- continue the above two steps if the prototypes have changed significantly;

\subsection{Segmentation}

Segmentation is a crucial part of an image classification method where the brain image is segmented to isolate different objects like gray matter, white matter, cerebral spinal fluid, skull, Tumor, etc., from each other and the background, as well as different Tumors, are labeled. In this work, MLS method is developed by combining optimal thresholding followed by watershed segmentation and morphological operations. The proposed MLS method is applied in which firstly, we used the optimal thresholding method that processes the input brain image using single-pixel by pixel value. The thresholding process for a gray level $(N)$ can be described as:

$$
\mathbf{f}_{n}(i, j)= \begin{cases}0 & \text { if } \mathbf{f}(i, j) \leq T \\ N-1 & \text { if } \mathbf{f}(i, j)>T\end{cases}
$$

where $T$ is the threshold value. To find the best thresholding value, the optimal thresholding process considers the histogram of an image to be a weighted sum of two or more probabilities densities. The threshold value is then set as the gray level that results in the smallest number of pixels being misclassified, i.e. background pixels being arranged in classes as foreground and foreground as background. The iterative self-organizing data analysis technique algorithm (isodata) $[14,15]$ is widely used to find threshold value in the optimal thresholding process that contains the below steps:

- threshold the image using the mean of the two peaks or the mean of all pixel values, $\left(T_{0}\right)$;

- calculate the new mean value of the pixels with a gray value less than or equal to the threshold, $\left(T_{0}\right)$, call it $\mu_{1}$, and the mean of the all pixels with a gray value greater than the threshold, $\left(T_{0}\right)$, call it $\mu_{2}$;

- compute the new threshold, $T_{i}=\left(\mu_{1}+\mu_{2}\right) / 2$, and threshold the image using the value, $T_{i}$;

- continue to compute the threshold value $T_{i}$, until $T_{i}-T_{i-1} \leq \Delta$; where $\Delta$, is the relative change in threshold value;

Then watershed segmentation is applied to separate multi-label regions that are close together of the thresholded objects and finally, the morphological operation is utilized precisely segment the defected area of Tumor. 


\subsection{Feature Extraction}

Feature extraction process extracts feature from the segmented brain Tumors and in the MLS-CNN process, the CNN method is used for feature extraction as it is now the go-to model on every image related problem. The main advantage of CNN compared to its predecessors is that it automatically detects the important features without any human supervision. Also, It can perform parameter sharing that enables us to run the CNN model on any device. Moreover, this approach requires less amount of data for faster training and it searches for the features at their base level. It consists of a series of special convolution with pooling operations followed by several fully connected layers. In this work, each convolution layer is designed using 16 filters of size 5. The basic architecture of the CNN model can be best described by the Figure 2.

CNN technique accepts an input image of size height $\times$ width $\times$ dimension where dimension indicates the image either RGB or grayscale, process it under certain categories. Technically, the image input layer of the CNN model passes the input data for training and testing through a series of convolution layers with filtering details, pooling, fully connected layers, and softmax function to classify an object with probabilistic values between 0 and 1 . Convolution layer extracts the features of an input image in which the convolution method preserves the relationship between pixels by learning image features. The number of filters deployed in the convolution layer allows more features discovered but with the cost of more training time. The pooling layer reduces the number of parameters by subsampling operation retaining important information. The fully connected layer that is similar to a neural network converted the feature map matrix into a vector. The features vector group together to create a model using a softmax operation that is used to classify as the desired object.

\subsection{Classification}

Classification is the final step of the image analysis method that involves sorting feature data in an image into separate classes. After segmenting a suspicious region, feature extraction and selection scheme are performed to extract the relevant information from the region; and a classification technique is used so that the best results are achieved, based on the available features and the Tumor classes. In this work, KSVM [16] is used as it can work clearly with non-linearly separable data in which the kernel function $(K)$ is defined as:

$$
K\left(\mathbf{x}, \mathbf{x}_{i}\right)=\phi^{\mathrm{T}}(\mathbf{x}) \phi\left(\mathbf{x}_{i}\right)
$$

where the training vectors $\mathbf{x}_{i}$ are mapped into a higher dimensional space by the function $\phi$ which is chosen based on the kernel used. Basically, the KSVM technique projects the data lower dimensions to linearly separable data in higher dimensions in such a way that data points belonging to different classes are allocated to different dimensions.

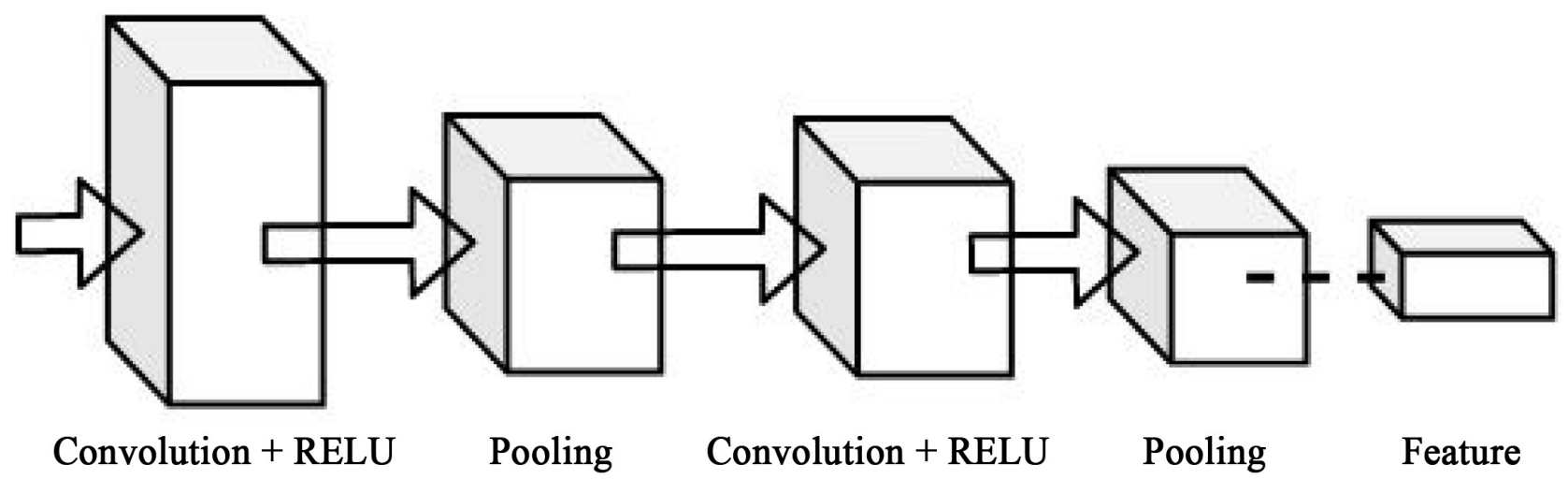

Figure 2. Block diagram of $\mathrm{CNN}$ architecture. 


\section{EXPERIMENTAL RESULTS}

The performance of the proposed MLS-CNN algorithm is evaluated using the brain MRI dataset which was downloaded from Havard Medical School [17]. For experiment evaluation, we created training and testing dataset that can be best described by the Table 1 .

In this experiment, we choose a total of 128 images for training and testing database in which $30 \%$ (38 images) data of all images were set to testing purposes and the rest of the $70 \%$ (90 images) images were set to the training dataset. For classification, the input image was preprocessed for reducing noise, smoothing and sharpening using Gaussian, high pass and median filtering techniques, respectively that show the effectiveness in the clustering and segmentation process. Then we applied our proposed MLS process to separate Tumor from the input image. Finally, the CNN technique was applied to extract features and classify the brain image as cancerous or non-cancerous Tumor using the features vector. The visual outputs of the basic steps of our proposed method for a cancerous and non-cancerous Tumor are shown in Figure 3 and Figure 4 respectively. From Figure 3, it is shown that the Tumor is easily classified as cancerous in which the segmented Tumor is clear and almost standalone. However for non-cancerous Tumor, the Tumor is not clear as there is very small difference with the tissues or other parts of the brain. Figure 4 is an example of a non-cancerous Tumor with yellow indicator shows the Tumor that is almost similar to the tissue of the brain.

For binary classification, KSVM is the most widely used technique to classify the object with appropriate accuracy. To validate the classification process, accuracy, precision and recall are used as qualitative assessments. The accuracy, precision and recall formula can be defined as [18]:

Table 1. Design process of brain MRI dataset with different types of Tumor.

\begin{tabular}{lccccccc}
\hline \multicolumn{3}{c}{ Training image set } & \multicolumn{5}{c}{ Testing Image set } \\
\hline \multicolumn{2}{l}{ Non-cancerous Tumor } & \multicolumn{2}{c}{ Cancerous Tumor } & \multicolumn{2}{c}{ Non-cancerous Tumor } & \multicolumn{2}{c}{ Cancerous Tumor } \\
\hline Diseases Name & No. & Diseases Name & No. & Diseases Name & No. & Diseases Name & No. \\
\hline $\begin{array}{l}\text { Alzheimer with } \\
\text { Agnosia }\end{array}$ & 12 & Glioma & 12 & $\begin{array}{l}\text { Alzheimer with } \\
\text { Agnosia }\end{array}$ & 05 & Glioma & 05 \\
Alzheimer & 11 & Meningioma & 11 & Alzheimer & 05 & Meningioma & 05 \\
Huntington & 11 & Metastatic & 11 & Huntington & 05 & Metastatic & 04 \\
Picks & 11 & Sarcoma & 11 & Picks & 04 & Sarcoma & 05 \\
& Total Images: 90 & & & Total Images: 38 & \\
\hline
\end{tabular}

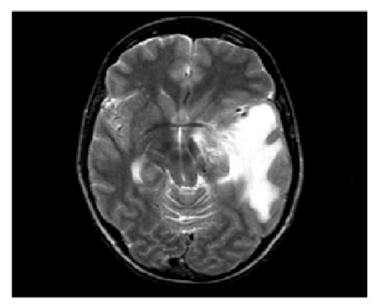

(a) Input image

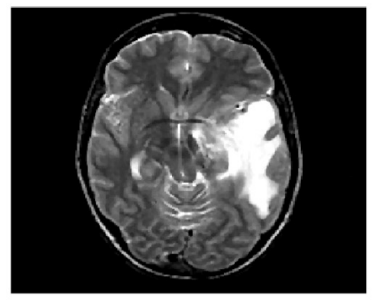

(b) Preprocessed

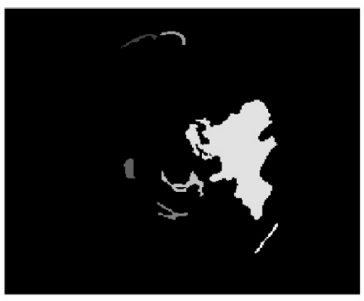

(c) Segmented Tumor

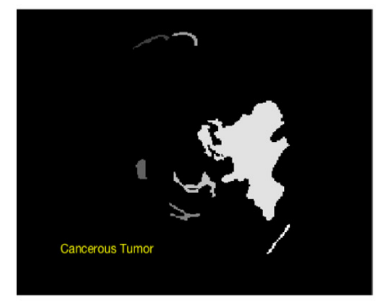

(d) Classified Tumor

Figure 3. Visual outputs of our proposed MLS-CNN based classification process for cancerous tumor. 


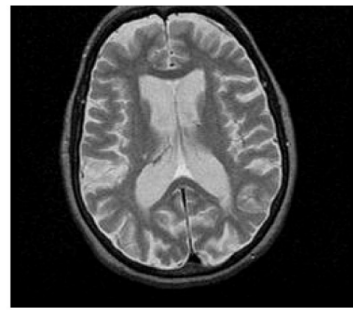

(a) Input image

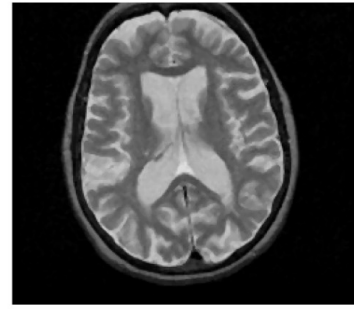

(b) Preprocessed

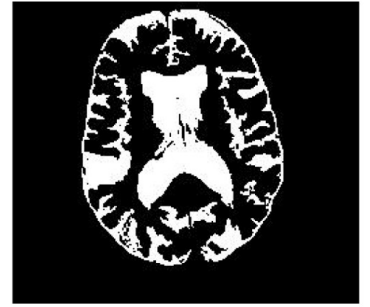

(c) Segmented Tumor

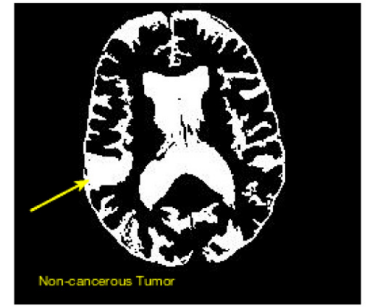

(d) Classified Tumor

Figure 4. Visual outputs of our proposed MLS-CNN based classification process for non-cancerous tumor.

$$
\begin{aligned}
& \text { accuracy }=\frac{\text { Number of correct predictions }}{\text { Total number of predictions }}, \\
& \text { precision }=\frac{\text { True positives }}{\text { true positives }+ \text { false positives }}
\end{aligned}
$$

and

$$
\text { recall }=\frac{\text { True positives }}{\text { true positives }+ \text { false negatives }}
$$

For all testing images, we measured accuracy, precision and recall value and the measurements of our algorithm is shown in Table 2.

For another assessment, a confusion matrix is computed and the corresponding confusion matrix is shown in Figure 5. In this figure, the first two diagonal cells show the number and percentage of correct classifications by the trained network. As shown in the above Figure, 18 images are correctly classified as non-cancerous. that corresponds to $47.4 \%$ of all 38 images. Similarly, 19 cases are correctly classified as cancerous and it corresponds to $50.0 \%$ of all images. One (1) of the cancerous images are incorrectly classified as non-cancerous which corresponds to $2.6 \%$ of all 38 images in which none of the non-cancerous images are incorrectly classified. Here, it is noted that since the early stage of cancerous Tumor is very close to the last stage of non-cancerous Tumor or vice-versa, the proposed system incorrectly identifies that Tumor. Out of 19 non-cancerous predictions, 18 (94.7\%) are correct and 1 (1.1\%) are wrong. Out of 19 cancerous predictions, $19(100 \%)$ are correct and $0(0 \%)$ are wrong. Out of 18 non-cancerous cases, $100 \%$ are correctly predicted as non-cancerous. Out of 20 cancerous cases, 19 (95.0\%) are correctly classified as cancerous and $1(2.6 \%)$ are classified as non-cancerous. Overall, $97.4 \%$ of the predictions are correct and $2.6 \%$ are wrong classifications.

\section{CONCLUSION}

In this paper, an efficient MLS-CNN model was proposed for Tumors detection and classification from the brain MRI image data set. To achieve the objectives of our algorithm, firstly we applied filtering techniques to reduce noise, sharpening and smoothing the image. Then we utilized the k-means clustering technique followed by the multilevel segmentation method to properly separate the Tumors from the brain MRI image. In this work, the proposed MLS method was developed combining the optimal thresholding and watershed segmentation algorithm followed by a morphological operation where the optimal threshold value was obtained by the isodata algorithm that improved the overall performance of the algorithm. Finally, CNN and KSVM algorithm was employed to extract the features and classify the cancerous or non-cancerous brain Tumors. Extensive experimental results have demonstrated that the use of CNN 
Table 2. Performance evaluation of the algorithm.

\begin{tabular}{ccc}
\hline Precision & Recall & Accuracy \\
\hline $94.8 \%$ & $100.0 \%$ & $97.4 \%$ \\
\hline
\end{tabular}

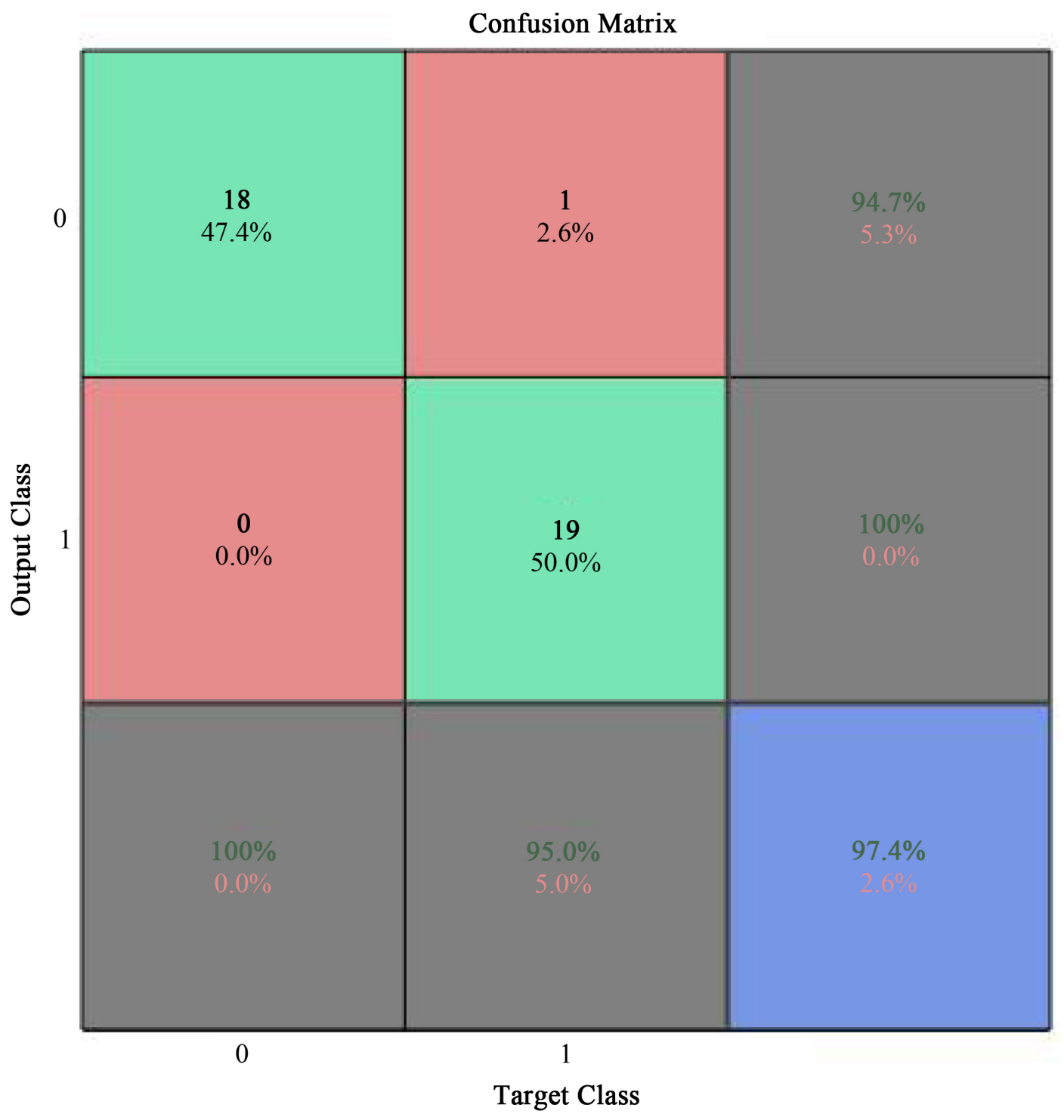

Figure 5. Confusion matrix of the algorithm for validation data set.

with the proposed MLS-CNN method results in a flexible and effective framework for Tumor detection and classification tasks. The proposed MLS-CNN method classifies the Tumor appropriately that was validated by using the accuracy, precision and recall value.

\section{ACKNOWLEDGEMENTS}

The authors thank the Department of Computer Science and Engineering of Dhaka University of Engineering \& Technology, Gazipur for providing research support to continue the research work. The authors also thank to the research \& extension department of Dhaka University of Engineering \& Technolo- 
gy, Gazipur for providing research fund to continue the work.

\section{CONFLICTS OF INTEREST}

The authors declare that they have no conflict of interest and this work does not contain any studies with human participants or animals performed by any of the authors.

\section{REFERENCES}

1. Gordillo, N., Montseny, E. and Sobrevilla, P. (2013) State of the Art Survey on MRI Brain Tumor Segmentation. Magnetic Resonance Imaging, 31, 1426-1438. https://doi.org/10.1016/j.mri.2013.05.002

2. Mohsen, H., El-Dahshan, E.-S., El-Horbarty, E.-S. and Salem, A.-B.M. (2017) Brain Tumor Type Classification Based on Support Vector Machine in Magnetic Resonance Images. Annals of Dunarea de Jos University of Galati, Fascicle II, No. 1, 75-88.

3. Pearson, K. (1901) On Lines and Planes of Closest Fit to Systems of Points in Space. The London, Edinburgh, and Dublin Philosophical Magazine and Journal of Science, 2, 559-572.

https://doi.org/10.1080/14786440109462720

4. Cortes, C. and Vapnik, V. (1995) Support-Vector Networks. Machine Learning, 20, 273-297. https://doi.org/10.1007/BF00994018

5. Abdullah, N. and Ngah, U.K. (2011) Improvement of MRI Brain Classification Using Principal Component Analysis. IEEE International Conference on Control System, Computing and Engineering, Penang, 25-27 November 2011, 557-561. https://doi.org/10.1109/ICCSCE.2011.6190588

6. Liu, F.Y. and Brown, M. (2019) Breast Cancer Recognition by Support Vector Machine Combined with Daubechies Wavelet Transform and Principal Component Analysis. In: Pandian, D., Fernando, X., Baig, Z. and Shi, F.Q., Eds., Proceedings of the International Conference on ISMAC in Computational Vision and Bio-Engineering, Springer International Publishing, Cham, 1921-1930. https://doi.org/10.1007/978-3-030-00665-5 177

7. Zhang, Y.D. and Wu, L.N. (2012) An MRI Brain Images Classifier via Principal Component Analysis and Kernel Support Vector Machine. Progress in Electromagnetics Research, 130, 369-388.

https://doi.org/10.2528/PIER12061410

8. Kaya, I.E., Pehlivanl, A.A., Sekizkarde, E.G. and Ibrikci, T. (2017) PCA Based Clustering for Brain Tumor Segmentation of T1w MRI Images. Computer Methods and Programs in Biomedicine, 140, 19-28.

https://doi.org/10.1016/j.cmpb.2016.11.011

9. Mathew, A.R. and Anto, P.B. (2017) Tumor Detection and Classification of MRI Brain Image Using Wavelet Transform and SVM. 2017 International Conference on Signal Processing and Communication, July 2017, 75-78. https://doi.org/10.1109/CSPC.2017.8305810

10. Abiwinanda, N., Hanif, M., Hesaputra, S.T., Handayani, A. and Mengko, T.R. (2019) Brain Tumor Classification Using Convolutional Neural Network. In: Lhotska, L., Sukupova, L., Lackovic, I. and Ibbott, G.S., Eds., World Congress on Medical Physics and Biomedical Engineering 2018, Springer, Singapore, 183-189. https://doi.org/10.1007/978-981-10-9035-6_33

11. Pereira, S., Pinto, A., Alves, V. and Silva, C. (2016) Brain Tumor Segmentation Using Convolutional Neural Networks in MRI Images. IEEE Transactions on Medical Imaging, 35, 1240-1251.

https://doi.org/10.1109/TMI.2016.2538465

12. Gu, J.X., Wang, Z.H., Kuen, J., Ma, L.Y., Shahroudy, A., Shuai, B., Liu, T., Wang, X.X. and Wang, G. (2015) Recent Advances in Convolutional Neural Networks. CoRR, abs/1512.07108.

13. Lloyd, S. (1982) Least Squares Quantization in PCM. IEEE Transactions on Information Theory, 28, 129-137. https://doi.org/10.1109/TIT.1982.1056489 
14. Dias Velasco, F.R. (1980) Thresholding Using the Isodata Clustering Algorithm. IEEE Transactions on Systems Man and Cybernetics, 10, 771-774. https://doi.org/10.1109/TSMC.1980.4308400

15. Ridler, T.W. and Calvard, S. (1978) Picture Thresholding Using an Iterative Selection Method. IEEE Transactions on Systems, Man, and Cybernetics, 8, 630-632. https://doi.org/10.1109/TSMC.1978.4310039

16. Cristianini, N. and Shawe-Taylor, J. (2000) An Introduction to Support Vector Machines and Other Kernelbased Learning Methods. Cambridge University Press, Cambridge. https://doi.org/10.1017/CBO9780511801389

17. Harvard Medical School. http://www.med.harvard.edu/aanlib

18. Powers, D.M.W. (2011) Evaluation: From Precision, Recall and F-Measure to ROC, Informedness, Markedness \& Correlation. Machine Learning Technologies, 2, 37-63. 